\title{
The X-ray Spectral Properties for Gamma-ray Loud Blazars
}

\author{
Ben-Zhong Dai \\ Department of Physics, Yunnan University, Kunming 650091, China
}

\begin{abstract}
We selected a sample of bright $\gamma$-ray $(\mathrm{E}>100 \mathrm{MeV})$ blazars whose X-rays in 0.1-10.0 keV band are observed by ROSAT and other Xray satellites, and make a detailed spectral analysis between the soft (0.1$2.0 \mathrm{keV})$ and hard band $(2.0-10.0 \mathrm{keV})$ as well as between X-ray and $\gamma$-ray properties using both un-weighted and weighted analysis methods. Our results indicate: (i) there are significant anti-correlations of the spectral indices between soft X-rays and $\gamma$-rays, and between hard X-ray and $\gamma$ ray of flat-spectrum radio quasars (FSRQs) and BL Lac objects; (ii) in the X-ray band, the correlation is confusing, there is positive correlation of the spectral indices between soft and hard X-ray band using weighted correlation analysis, but this correlation is negative using un-weighted correlation analysis.
\end{abstract}

\section{Introduction}

Blazars (including FSRQs and BL Lac objects) are highly variable sources characterized by non-thermal emission that dominates from the radio to the $\gamma$-rays. This emission is supposedly due to a relativistic jet seen at a small angle to the line of sight (Blandford \& Rees 1978). In the third catalog of EGRET, there are 66 confirmed blazars (Hartman et al. 1999, however, recent analysis of Mattox et al. (2001) pointed out that 46 objects are confirmed). Very high energy gamma-rays from a few nearby sources have been detected up to the $\mathrm{TeV}$ energies with ground-based Cherenkov telescopes (see review of Catanese \& Weeks 1999). Gamma-ray observations have revealed the remarkable feature that the overall Spectral Energy Distribution (SED) of a blazar shows two distinct components in the $\nu-\nu F_{\nu}$ representation, typically characterized by their peak energies. The low energy component peaks from $\mathrm{mm}$ to the X-rays, while the high energy peaks at $\mathrm{GeV}-\mathrm{TeV}$ energies. The $\mathrm{X}$-ray observation allows us to estimate the minimum of the SEDs and hence to derive physical parameters to constrain the theoretical models. Further constraints on the emission mechanisms can be obtained from the variability pattern and the slope of the X-ray spectrum (Ulrich, Maraschi, \& Urry 1997)

To study the spectral indices properties, we have collected data for all blazars having available spectral information both in the soft and hard X-ray band and $\gamma$-ray band by using following selection: (i) 46 blazars with radio identifications which have high probability of being correct (Mattox, Hartman, \& Reimer 2001), and (ii) the sources have available spectral information both 

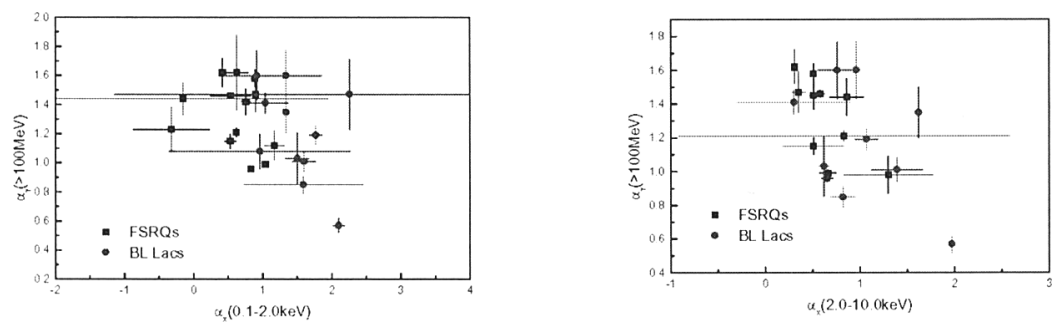

Figure 1. The spectral relations between gamma-ray and X-ray. The left panel is for $\alpha_{\gamma}$ against $\alpha_{x}(0.1-2.0 \mathrm{keV})$ and the right panel for $\alpha_{\gamma}$ against $\alpha_{x}(2.0-10.0 \mathrm{keV})$

in the soft and hard X-ray bands and $\gamma$-ray band. Such a selection yields 26 bright $\gamma$-ray blazars. The $\gamma$-ray energy spectral indices are from Hartman et al. (1999). The X-ray data are from Donato et al. (2001) and references therein. For the soft band (0.1-2.0 keV), most of the results come from ROSAT, while for the 2.0-10.0 keV band the results are gathered from the EXOSAT, ASCA and BeppoSAX satellites (see detail in Donato et al. 2001). For most of the objects, there exist several X-ray observations. In these cases, we have chosen the X-ray flux and spectral slope according to the following criteria : (1) the X-ray flux (1 $\mathrm{keV}$ ) and soft spectral index referring to the same observation, (2) some sources have been observed many times either by the same and/or by different satellites, the results of the most recent analysis are preferred. The spectral indices are written $f_{\nu} \propto \nu^{-\alpha}$.

\section{Results}

The mean $\gamma$-ray and X-ray spectral indices are $1.49 \pm 0.67,1.06 \pm 0.16,1.20 \pm 0.12$ for BL Lacs (11 sources) in $\left\langle\alpha_{0.1-2.0 \mathrm{keV}}\right\rangle,\left\langle\alpha_{2.0-10.0 \mathrm{keV}}\right\rangle$ and $\left\langle\alpha_{\gamma}\right\rangle$ respectively, $0.61 \pm 0.29,0.64 \pm 0.28,1.31 \pm 0.09$ for for FSRQs (15 sources). We find a significantly different distributions of soft and hard X-ray band spectral indices between BL Lac objects and FSRQs. We give the probability, according to the Kolmogorov-Smirnov (KS) test, that two distributions are drawn from the same parent population. The KS test gives a probability $\mathrm{P}=0.71$ for $\alpha_{\gamma}$, $1.2 \times 10^{-4}$ for $\alpha_{0.1-2.0 \mathrm{keV}}$, and 0.11 for $\alpha_{2.0-10.0 \mathrm{keV}}$. The obtained results indicate that the both the soft and hard X-ray spectra of BL Lac objects are steeper than FSRQs, as first suggested by Worral \& Wilkes (1990). Comastri et al. (1997) found the similar spectral property trend considering only soft X-ray band.

When using the un-weighted correlation analysis using a nonparametric Spearman rank test, we find significant anti-correlations in all three band. The results are shown in Table 1. The anti-correlation between the two spectral indices $\alpha_{0.1-2.0 \mathrm{keV}}$ and $\alpha_{\gamma}$ is consistent with the results obtained by Comastri et al. (1997) when both FSRQs and BL Lac objects are considered. The available spectral indices in our sample are observed with different instruments in different time. So, we need consider the different observation errors in statisti- 


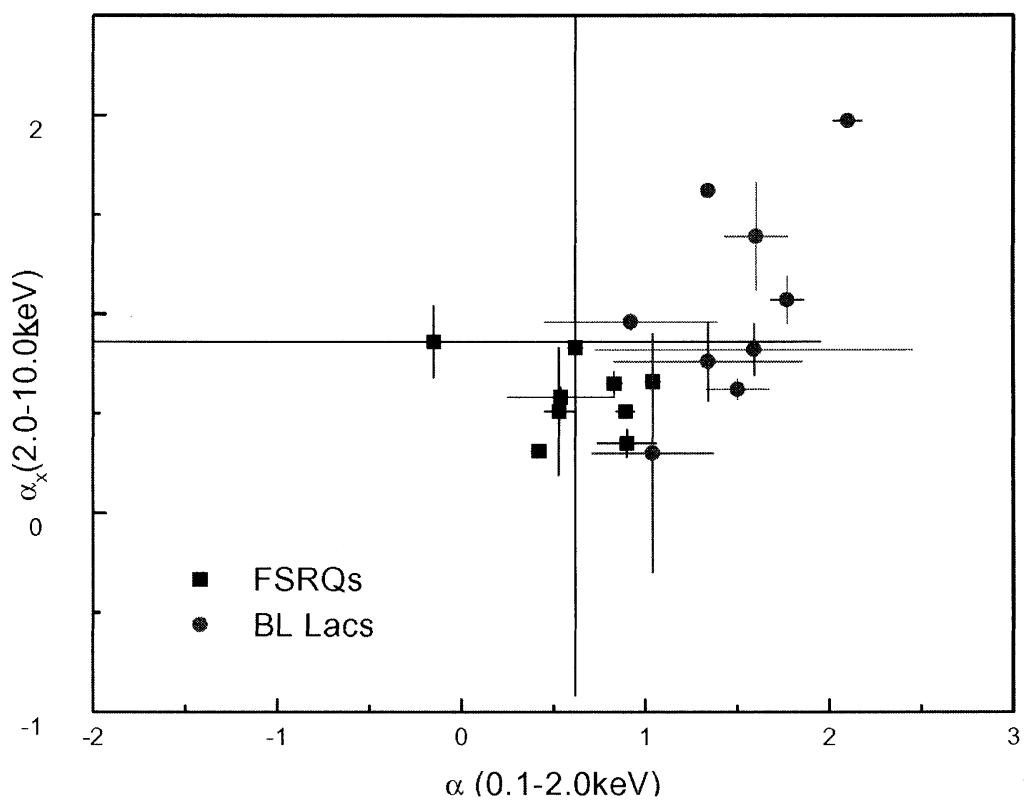

Figure 2. The plot of $\alpha_{x}(0.1-2.0 \mathrm{keV})$ against $\alpha_{x}(2.0-10.0 \mathrm{keV})$

cal analysis for reflecting the real physical properties. The weighted correlation analysis results using a nonparametric Spearman rank test are shown in table 1. The weighted anti-correlation between the two spectral indices $\alpha_{0.1-2.0 \mathrm{keV}}$ and $\alpha_{\gamma}(99.9 \%)$ is higher than the un-weighted results using same statistical analysis method.The most difference between the two results is that the weighted correlation is positive but the anti-correlation in un-weighted result for the correlation $\alpha_{0.1-2.0 \mathrm{keV}}$ vs $\alpha_{2.0-10.0 \mathrm{keV}}$.

FSRQs and BL Lac objects occupy two different regions both in the $\alpha_{0.1-2.0 \mathrm{keV}}-\alpha_{\gamma}$ and $\alpha_{0.1-2.0 \mathrm{keV}}-\alpha_{2.0-10.0 \mathrm{keV}}$ plane (see left panel in Figure 1 and Figure 2). A flat soft X-ray spectrum is thus associated with a steep $\gamma$-ray slope for FSRQs, while the reverse is true for BL Lac objects. In the X-ray band, flat soft X-ray spectrum is associated the flat hard X-ray slope for FSRQs

Table 1. The correlation analysis results

\begin{tabular}{lcrr}
\hline Correlation & BL Lacs (N) $(p)$ & FSRQs (N) $(p)$ & \multicolumn{1}{c}{ all blazars (N) $(p)$} \\
\hline \multicolumn{4}{c}{ The un-Weighted Correlation Results } \\
\hline$\alpha_{\text {soft vs } \alpha_{\text {hard }}}$ & $0.61(9)(93.2 \%)$ & $-0.35(9)(65 \%)$ & $-0.59(18)(99 \%)$ \\
$\alpha_{\text {soft vs }} \alpha_{\gamma}$ & $-0.41(11)(78 \%)$ & $-0.22(13)(52 \%)$ & $-0.38(24)(94 \%)$ \\
$\alpha_{\text {hard }}$ vs $\alpha_{\gamma}$ & $-0.49(9)(82 \%)$ & $-0.59(11)(94.4 \%)$ & $-0.55(20)(98.7 \%)$ \\
\hline \multicolumn{4}{c}{ The Weighted Correlation Results } \\
\hline$\alpha_{\text {soft vs } \alpha_{\text {hard }}}$ & $0.63(9)(93 \%)$ & $0.55(9)(88 \%)$ & $0.87(18)(>99.99 \%)$ \\
$\alpha_{\text {soft }}$ vs $\alpha_{\gamma}$ & $-0.94(11)(>99.99 \%)$ & $-0.58(13)(96.1 \%)$ & $-0.63(24)(99.9 \%)$ \\
$\alpha_{\text {hard }}$ vs $\alpha_{\gamma}$ & $-0.54(9)(86 \%)$ & $-0.50(11)(89 \%)$ & $-0.52(20)(98.1 \%)$ \\
\hline
\end{tabular}


and the soft X-ray spectral indices increase with the hard X-ray spectral indices increase for BL Lac objects. In the $\alpha_{2.0-10.0 \mathrm{keV}}-\alpha_{\gamma}$ plane, the two objects can not be separated (see right panel in Figure 1). For FSRQs, the X-ray emission is mainly due to the inverse Compton scattering because of the flatter spectral index and spectral slope. It should be pointed out that the steepest indices correspond to HBLs $\left(\alpha_{0.1-2.0 \mathrm{keV}}=1.72\right.$ and $\left.\alpha_{2.0-10.0 \mathrm{keV}}=1.80\right)$, where the Xrays are entirely dominated by the steep tail of the synchrotron emission. The correlation between $\alpha_{2.0-10.0 \mathrm{keV}}$ and $\alpha_{\gamma}$ for all blazars show that the FSRQs and BL Lacs are mixture (Figure 2). This also suggests that in the hard X-ray, the emission is dominated by the same radiation mechanism for FSRQs and BL Lacs with inverse Compton process.

Acknowledgments. We acknowledge support from the National Natural Science Foundation of China under the grant no. 10263001 and the Natural Science Foundation of Yunnan Province (2000A004M).

\section{References}

Blandford R. D.,\& Rees M. J. 1978, in Pittsburgh Conf. on BL Lac Objects, ed.A. M. Wolfe (Pittsburgh: Univ. Pittsburgh Press), 328

Catanese M., \& Weeks T. C. 1999, PASP, 111, 1193

Comastri A., Fossati G., Ghisellini G. \& Molendi S. 1997, ApJ, 480, 534

Donato D., Ghisellini G., Tagliaferri G., \& Fossati G. 2001, A\&A, 375, 739

Hartman R. C., Bertsch D. L., Bloom S. D. et al. 1999, ApJS, 123, 79

Mattox J. R., Hartman R. C. \& Reimer O., 2001, ApJS, 135, 155

Ulrich M.-H., Maraschi L., \& Urry C.M. 1997, ARA\&A, 35, 445

Worral D. M., \& Wilkes B. J. 1990, ApJ, 360, 396 